\title{
Previously Unreported COL7A1 Mutation in a Somali Patient with Dystrophic Epidermolysis Bullosa
}

\author{
Valeria Venti ${ }^{a}$ Bruna Scalia $^{a}$ Alessandra Sauna $^{a}$ Maria Rita Nasca $^{b}$ \\ Pierluigi Smilari $^{a} \quad$ Andrea D. Praticò $^{a}$ Agata Fiumara $^{a} \quad$ Xena G. Pappalardo ${ }^{c}$ \\ Piero Pavone \\ a Section of Pediatrics and Child Neuropsychiatry, Department of Clinical and Experimental Medicine, University of

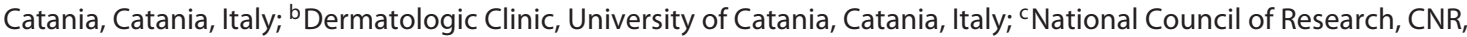 \\ Institute for Research and Innovation in Biomedicine (IRIB) Unit of Catania, Catania, Italy
}

\section{Established Facts}

- Dystrophic epidermolysis bullosa (DEB) includes variants inherited in both autosomal dominant or autosomal recessive manner.

- All subtypes are mainly associated with mutations in the COL7A1 gene encoding type VII collagen, a major stabilizing molecule of the dermoepidermal junction.

\section{Novel Insights}

- The variant c.6797G> (exon 86) in the COL7A1 gene found in the patient was predicted to alter the correct assembly of the collagenic domain of type VII, thus causing a severe phenotype of DEB.

- This c.6797G>T variant has not previously been reported to be associated with DEB.

\section{Keywords}

COL7A1 variant - Cutaneous disorders · Dystrophic epidermolysis bullosa - Epidermolysis bullosa .

Pathogenetic variants

\section{Abstract \\ Epidermolysis bullosa (EB) encompasses a group of inherit- able skin disorders characterized by various degrees of epi- thelial fragility that lead to cutaneous and mucosal blistering following negligible mechanical traumas. These disorders are clinically and genetically heterogeneous, ranging from}

mild skin involvement to severe disabling conditions with associated manifestations affecting the gastrointestinal and vesico-urinary tracts. EB may be classified into 4 main categories: simplex, junctional, dystrophic, and Kindler syndrome. Clinically, EB may present as syndromic or nonsyndromic forms. EB subtypes have mainly reported a number of mutations in the candidate COL7A1 gene encoding type $\mathrm{VII}$ collagen, a major stabilizing molecule of the dermoepidermal junction. Herein, we report a Somali girl with dystrophic EB who showed a previously unreported missense variant c.6797G > T in exon 86 in COL7A1.

(c) 2019 S. Karger AG, Base
KARGER

(C) 2019 S. Karger AG, Basel

E-Mail karger@karger.com

www.karger.com/msy
Piero Pavone, $\mathrm{PhD}$

Section of Pediatrics and Child Neuropsychiatry, Department of Clinical and Experimental Medicine University of Catania

Via S. Sofia 78, IT-95123 Catania (Italy)

E-Mail ppavone@unict.it 
Epidermolysis bullosa (EB) encompasses a group of inheritable skin disorders characterized by various degrees of epithelial fragility that lead to cutaneous and mucosal blistering following negligible mechanical traumas. These disorders are clinically heterogeneous ranging from mild skin involvement to severe disabling conditions with associated manifestations affecting the gastrointestinal and vesico-urinary tracts as well as extremities. Nail dystrophy and alopecia, frequent microbial superinfections, and severe scarring can result from aberrant healing with development of pigmentary changes, milia, tissue adhesions, and permanent ocular damage. Multisystem involvement may result in significant morbidity characterized by chronic inflammation, malnutrition, growth retardation, failure to thrive, osteoporosis, anemia, cachexia, and other symptoms related to specific organ damage [Laimer et al., 2015; Rashidghamat and McGrath, 2017; Has et al., 2018].

Blistering is pathogenetically related to genetically driven molecular aberrations impairing the integrity and function of intraepidermal or dermoepidermal structures essential for intraepithelial intercellular adhesion or for cell-substrate anchoring in the basal membrane zone. The level of cleavage can be assessed by diagnostic transmission-electron microscopy and/or immune epitope mapping. Thus, EB may be classified into 4 main categories: simplex (tissue separation occurs within the basal layer of the epidermis), junctional (tissue separation occurs within the lamina lucida of the basal membrane), dystrophic (tissue separation occurs within the upper papillary dermis just below the lamina densa of the basal membrane), and Kindler syndrome (tissue separation occurs at different levels during wound healing as a result of defective actin cytoskeleton attachment) [Fine et al., 2014; Laimer et al., 2015; Has et al., 2018]. In addition, EB has been recently classified in syndromic and nonsyndromic forms [Vahidnezhad et al., 2019a]. Syndromic EB encompasses a group of cutaneous involvement in association with extracutaneous clinical manifestations with a distinct set of genetic mutations [Vahidnezhad et al., 2019a]. Dystrophic EB (DEB) includes variants inherited in both autosomal dominant (OMIM 131750 and 131800) or autosomal recessive manner (OMIM 226600).

Molecular studies have shown that DEB subtypes are mainly associated with mutations in the COL7A1 gene encoding type VII collagen, a major stabilizing molecule of the dermoepidermal junction [Kon et al., 1998; Rashidghamat and McGrath, 2017; Has et al., 2018; Yenamandra et al., 2018]. The gene is located in 3p21.1 and consists of 118 coding exons. More than 841 mutations of

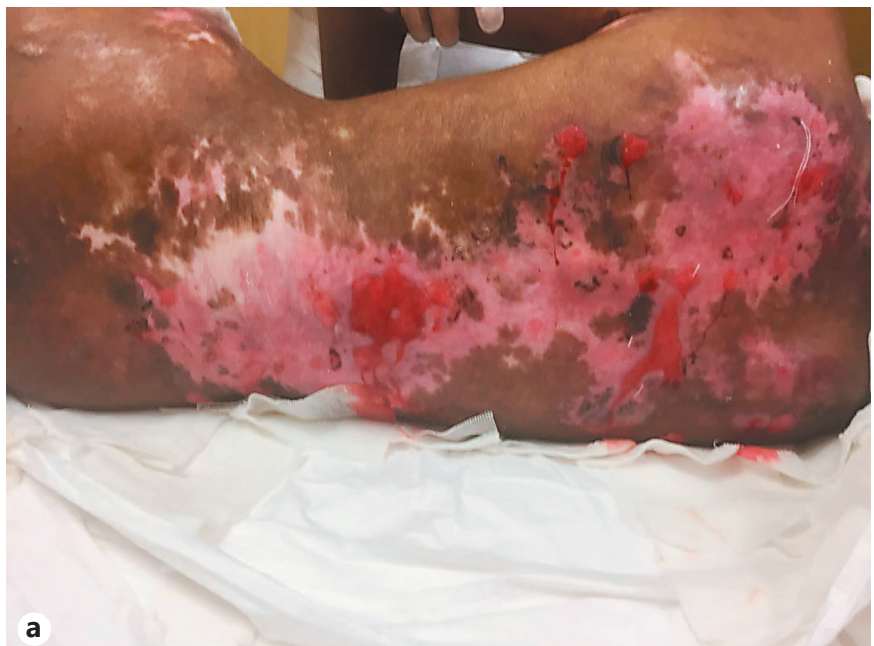

a

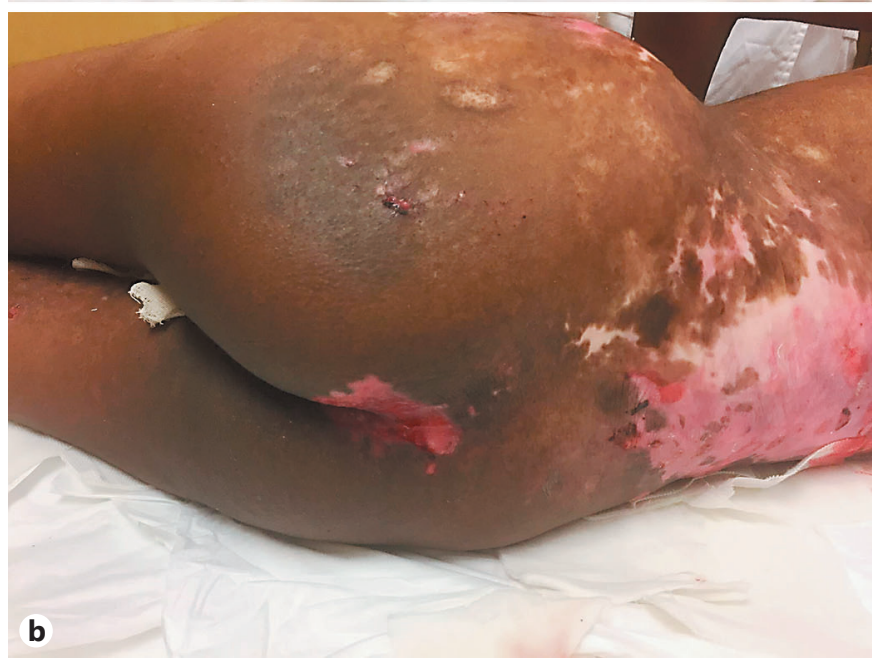

Fig. 1. a Photograph of skin lesions on the girl's back. b Photograph indicating mild improvement of skin lesions on the lower back a week after the onset of the treatment.

this gene have been identified so far, which are usually family specific and lead to variable structural and functional impairment of anchoring fibrils [Laimer et al., 2015; Rashidghamat and McGrath, 2017; Has et al., 2018].

Herein, we report a Somali girl with DEB associated with a previously unreported COL7A1 mutation based on comparisons with the DEB Patient Registry (http://www. deb-central.org) and other current resources for human variation data in public and commercial resources.

\section{Case Report}

The 12-year-old girl, a refugee from Somalia, came to our observation on July 2017, a few days after landing at Augusta (Italy) harbor. At admission, she was accompanied by an unrelated care- 
giver from the same country. The young girl did not provide news about her parents, but she mentioned her maternal grandmother being affected by a cutaneous disorder, who probably deceased due to condition-related complications. The girl's weight was $30 \mathrm{~kg}$ (25th percentile), height $136 \mathrm{~cm}$ (3th percentile), and her head circumference was $54 \mathrm{~cm}$ (50th percentile). She reported the occurrence of widespread skin erosions since her early years of life with variably sized blisters arising after minimal traumas and rapidly evolving into painful ulcers; these slowly resolved with severe scarring. At admission, physical examination showed multiple and large erosions mainly located to large axillary and inguinal folds but also extending from the neck to the dorsal region (Fig. 1a). Some of these lesions showed superinfected crusting or deepening into chronic ulcers. These lesions were surrounded by atrophic and dyschromic skin resembling postburn-injured areas and associated with sclerotic scar retractions deep in the inguinal and axillary fold effectively limiting limb mobility. She also had dystrophic nails and teeth with sparse caries and dental loss. Further neurological and ophthalmological examinations as well as abdominal ultrasound assessment were unremarkable.

A diagnostic algorithm was applied to rule out infectious, autoimmune, hematologic, metabolic, traumatic, genetic, or drug-induced causes for cutaneous blistering. Routine laboratory studies showed hypoalbuminemia $(2.27 \mathrm{~g} / \mathrm{dL})$ and sideropenic anemia $(\mathrm{Hb}$ $9.4 \mathrm{~g} / \mathrm{dL}$, serum iron $15 \mu \mathrm{g} / \mathrm{dL}$, ferritin $20 \mu \mathrm{g} / \mathrm{L}$ ). Skin (inguinal, axillary) and mucosal (nasal, pharyngeal, and auricular) swabs yielded Staphylococcus aureus sensitive to beta-lactam antibiotics. A Tzanck smear showed abundant neutrophils and a few lymphocytes with occasional keratinocytes devoid of morphological alterations. Analysis on the specimen taken by a punch biopsy from perilesional skin in the lower abdominal area showed subepidermal blistering containing traces of red blood cells and granulocytes with vascular ectasia, mild lymphohistiocytic infiltrate in the superficial dermis, and negative direct immunofluorescence findings (absence of IgG, IgA, IgM, or C3 deposits). The patient was managed with intravenous fluid and albumin infusions, antibiotic therapy with parenteral ceftriaxone, oral iron supplementation, and topical agents as well as nonadhesive dressings to promote wound healing with consistent but partial clinical improvement (Fig. 1b).

\section{Materials and Methods}

The mutational analysis for a diagnosis of DEB subtype was performed by isolating genomic DNA from blood leukocytes and subjected to 72 separate PCR amplifications using LightCycler $480^{\circledR}$ (Roche Life Science) to investigate all 118 COL7A1 exons and their flanking intronic sequences. PCR products were subjected to heteroduplex scanning utilizing conformation sensitive gel electrophoresis (CSGE) [Ganguly et al., 1993] and determined by direct sequencing on ABI Prism ${ }^{\circledR} 3100$ Genetic Analyzer (Thermo Fisher Scientific) according to the manufacturer's protocol. DNA sequence and fragment analysis was compared to the reference sequence from the NCBI Entrez Nucleotide database (NM_000094, L23982) using the manufacturer's software data analysis. To detect homozygous mutations, the PCR products generated from the proband were mixed with PCR products of control prior to CSGE. Whenever possible, the validation of a mutation was carried out either by restriction enzyme digestion or by allele-specific oligomer hybridization as described [Christiano et al., 1997].

\section{Results}

Sequencing of the entire COL7A1 gene revealed a homozygous single-base missense variant c.6797G $>\mathrm{T}$ in exon 86, corresponding to a non-glycine amino acid substitution (p.Gly2266Val) (Fig. 2a), not previously reported in the online DEB register database and in the most important genome variations repositories such as HGMD (www.hgmd.cf.ac.uk/), ClinVar (https://www.ncbi.nlm. nih.gov/clinvar/), 1000 Genomes (https://www.internationalgenome.org/category/variants/), Mastermind (https://mastermind.genomenon.com/), ExAc (http:// exac.broadinstitute.org/gene/), gnomAD (https://gnomad.broadinstitute.org/), and Global Variome shared LOVD (https://databases.lovd.nl/shared/genes) (Fig. 2b). Of note, most common mutations in dominant-inherited form are glycine substitutions in the collagenous domain of COL7A1 [Dang and Murrell, 2008]. Recessive forms of DEB are either homozygous or heterozygotes mutations carrying different mutations in the COL7A1 gene, known as "compound heterozygosity," leading to premature termination codons [Fine et al., 2014]. In order to investigate the effect of the protein variation, we performed a computational prediction using a bioinformatic tool, PROVEAN (http://provean.jcvi.org/about.php). According to PROVEAN score prediction (using default cutoff at -2.5 ), the G2266V variant is considered to be deleterious (output value of -6.319). Additional secondary protein structure prediction (http://npsa-pbil.ibcp.fr, http://bioinf. cs.ucl.ac.uk/psipred/) indicated that the G2266V mutation caused significant structural changes in collagen VII, from coil to sheet, arguing for a functional relevance of this variant.

\section{Discussion}

The clinical spectrum of DEB is wide but commonly severe, associated with significant morbidity that profoundly affects functional abilities and quality of life. Anchoring fibril defects lead to the development of cutane-

Fig. 2. a Schematic view of the COL7A1 gene modified from UCSC Genome Browser. Red arrow indicates the location of the novel missense variant c.6797 G>T in the exon 86 corresponding to a non-glycine amino acid substitution (p.Gly2266Val). b Image modified from UCSC Genome Browser showing genetic variations identified in the exon 86 of COL7A1. Data tracks of HGMD, ClinVar, LOVD, dbSNP, ExAC, gnomAD are indicated in bold red on the left.

(For figure see next page.)
Venti/Scalia/Sauna/Nasca/Smilari/ Praticò/Fiumara/Pappalardo/Pavone 





ous mechanobullous subepidermal blisters that heal with extensive scarring and milia formation. Severe and repeated acral blistering results in chronic ulcerations, synechiae, and joint contractures. Mitten-hand and foot deformities are frequently reported and indicated as a pathognomonic phenotype in DEB [Vahidnezhad et al., 2019a]. Altered genetic collagen VII expression also involves other epithelial tissues besides the skin affecting the gastrointestinal, respiratory, and urogenital tracts. These include oral mucosal blistering with fusion of the tongue to the floor of the mouth and progressive reduction of the size of the oral cavity (ankyloglossia, microstomia), esophageal erosions and strictures with severe dysphagia and malnutrition, anal strictures with coprostasis, and genitourinary involvement with urinary retention. Abnormalities of the appendages (scarring alopecia, dystrophic nails), increased risk of squamous cell carcinoma development, and ocular lesions (symblepharon, corneal erosions and opacities, visual loss) are also common. Thus, DEB is a multisystem disease and one of the most devastating subtypes of EB [Laimer et al., 2015]. Blistering and erosions affecting the whole body may be present as early as the neonatal period. The course involves chronic relapse due to recurrent hemorrhages, anemia, malnutrition, and consequent vitamin and mineral deficiencies. These features frequently reduce growth in young children and delays puberty. The lifetime risk of aggressive squamous cell carcinoma - usually arising in chronic wounds - is higher than $90 \%$ by the age of 55 years [Has et al., 2015; Laimer et al., 2015; Rashidghamat and McGrath, 2017].

The clinical and histopathological features observed in the patient were consistent with a diagnosis of inherited nonsyndromic DEB. Skin fragility, poor wound healing, and nail alterations had been present since earlier in life, while at present time, ocular and gastrointestinal alterations as well as hand and foot deformities were not observed.

Molecular analysis has offered important insights into the pathogenesis of DEB and revolutionized the modern approach to its diagnosis. Mutations in the COL7A1 gene located in the short arm of chromosome 3p21.1 are known to cause defects in the anchoring fibrils that result in a sublamina densa separation typical of DEB. The causative mutation of DEB may consist of single nucleotide substitutions, deletions, or insertions. This may result in premature termination codons, glycine substitutions, missense mutations, or splicing errors [Kon et al., 1998, Dang and Murrell, 2008]. Genetic heterogeneity with a different type, number, and location of the mutations may cause variable quantitative (complete absence, reduction) or qualitative (structural defects with loss of function) alterations of anchoring fibrils resulting in significant clinical variability [Laimer et al., 2015]. For instance, glycine substitutions within the central triple helix domain lead to expression of full-length collagen VII molecules with impaired functionality. These anomalies are usually associated with milder clinical expression [Dang and Murrell, 2008]. Multiple mutations may result in a similar phenotype, but alternatively, the same mutation may cause a broad phenotypic variability [Yenamandra et al., 2018]. This occurs because genotype-phenotype correlations are further complicated by secondary epigenetic and environmental factors that may influence disease severity [Dang and Murrell, 2008; Laimer et al., 2015]. The nature of mutations in COL7A1 and their positions are maintained to be correlated with the severity of the DEB phenotype [Dang and Murrell, 2008].

The susceptibility of type VII collagen to secondary proteolysis may be variable according to the different mutations [Dang and Murrell, 2008; Yenamandra et al., 2018]. Moreover, the expression of the type VII collagen gene can also be modulated by transforming growth factor beta, which is known to promote tumor development [Has et al., 2018; Kiritsi and Nyström, 2018]. Each EB subtype is known to arise from mutations within the genes encoding for several different proteins, each of which is intimately involved in the maintenance of keratinocyte structural stability or adhesion of the keratinocyte to the underlying dermis.

An advanced mutational analysis by combined Sanger sequencing and gene-targeted next generation sequencing, led by Vahidnezhad et al. [2017], was performed in 91 EB patients representing 91 families with a total of 133 affected individuals. By this explorative approach, 72 pathogenic or likely pathogenic variants were identified in 68 families in 11 distinct genes. Notably, among 72 mutations, 35 (48.6\%) were previously unreported. Identification of mutations in different genes allowed Vahidnezhad et al. [2017] to predict the EB subtypes in 76 out of 91 families, performing the first molecular taxonomy of EB.

More recently, a novel target protein encoded by the procollagen-lysine, 2-oxoglutarate 5-dioxygenase 3 gene (PLOD3), has been implicated as a causal factor of autosomal recessive DEB [Vahidnezhad et al., 2019a]. The PLOD3 mutation encoding lysyl hydroxylase 3 (LH3) was identified by whole-exome sequencing combined with genome-wide homozygosity mapping, and no mutations in COL7A1 were detected with the level of LH3 markedly reduced in the skin and the fibroblast cultures [Vahidnezhad et al., 2019b]. Furthermore, pathogenic variants in PLOD3 have been associated in a clinically distinct Stick-
Venti/Scalia/Sauna/Nasca/Smilari/ Praticò/Fiumara/Pappalardo/Pavone 
ler-like syndrome with vascular complications [Ewans et al., 2019].

In this study, we report the characterization of a novel homozygous single-base missense variant c.6797G $>\mathrm{T}$ in exon 86 of the COL7A1 gene in a young girl from Somalia, resulting in G2266V, a glycine missense mutation located in the triple helical region (Q02388; region: 1254-2784) of the gene. This mutation had not been previously reported on DEB registry and according to other current resources for human variation data in public and commercial resources (Fig. 2b).

This variant was predicted to alter the correct assembly of the collagenic domain of type VII collagen related to a severe phenotype of DEB as depicted by clinical features and protein interactions prediction (PROVEAN analysis; data non shown). Actually, glycine substitutions within the triple helical domain are typically involved in autosomal dominant DEB. This suggests that G2266V may lead to alter the protein conformation of the triple helix folding in the glycine residues [Kon et al., 1998; Dang and Murrell, 2008; Vahidnezhad et al., 2017; Saeidian and Youssefian, 2018; Saeidian et al., 2018; Yenamandra et al., 2018].

Early diagnosis of DEB is essential to prevent and/or minimize tissue damage via adequate clothing and semiocclusive, nonadhesive wound dressings. Patient care includes symptomatic control of pain and itch, treatment of microbial infections with antiseptics and antibiotics, correction of fluid and electrolyte imbalances as well as treatment of malnutrition with fluid infusions and supplements. A multidisciplinary cooperation with plastic surgeons is also advisable to manage less or more frequent complications such as squamous cell carcinoma development on troublesome areas by local excision or longstanding, nonhealing ulcerated wounds, contractures, and limb deformities by autologous and allogenic skin grafting, cultured epidermal autografts, and allogenic cultured dermal substitutes [Laimer et al., 2015; Rashidghamat and McGrath, 2017; Kiritsi and Nyström, 2018].
Though a cure for EB remains elusive, recent data on animal models and initial human clinical trials have raised the expectations of patients, clinicians, and researchers that disease modification and improved quality of life are feasible goals [Li et al., 2017; Rashidghamat and McGrath, 2017; Kiritsi and Nyström, 2018; Nakayama et al., 2018]. Preliminary studies using hematopoietic stem cells and mesenchymal stromal/stem cells, gene editing/ engineering, and clinical application of inducible pluripotent stem cells have produced promising results [Rashidghamat and McGrath, 2017]. Advancing knowledge in the use of gene therapy and targeted genome-editing techniques - coupled with the development of induced pluripotent stem cells - is expected to allow and extend the use of autologous therapies derived from patient-specific renewable populations of cells [Bremer et al., 2016; Kiritsi and Nyström, 2018].

In this regard, the detection and analysis of the individual genetic mutation is an essential step to plan the best individual treatment strategy and identify patients who could potentially benefit from tailored experimental genetic approaches.

\section{Acknowledgment}

We wish to thank the American Manuscript Editors (USA) for editing the manuscript.

\section{Statement of Ethics}

Informed consent was obtained from the caregiver prior to investigation. The study was approved by the Ethical Board of the University of Catania, Italy.

\section{Disclosure Statement}

The authors have no conflicts of interest to declare.

\section{References}

\footnotetext{
Bremer J, Bornert O, Nyström A, Gostynski A, Jonkman MF, et al: Antisense oligonucleotide-mediated exon skipping as a systemic therapeutic approach for recessive dystrophic epidermolysis bullosa. Mol Ther Nucleic Acids 5:e379 (2016).

-Christiano AM, Amano S, Eichenfield LF, Burgeson RE, Uitto J. Premature termination codon mutations in the type VII collagen gene in recessive dystrophic epidermolysis bullosa result in nonsense-mediated mRNA decay and absence of functional protein. J Invest Dermatol 109:390-394 (1997).
}

Dang N, Murrell DF: Mutation analysis and characterization of COL7A1 mutations in dystrophic epidermolysis bullosa. Exp Dermatol 17: 553-568 (2008).

Ewans LJ, Colley A, Gaston-Massuet C, Gualtieri A, Cowley MJ, et al: Pathogenic variants in PLOD3 result in a Stickler syndrome-like connective tissue disorder with vascular complications. J Med Genet 56:629-638 (2019).
Dystrophic Epidermolysis Bullosa in a Somali Patient
Mol Syndromol 2019;10:332-338 DOI: $10.1159 / 000504210$ 
Fine JD, Bruckner-Tuderman L, Eady RA, Bauer EA, Bauer JW, et al: Inherited epidermolysis bullosa: updated recommendations on diagnosis and classification. J Am Acad Dermatol 70:1103-1126 (2014).

Ganguly A, Rock MJ, Prockop DJ: Conformationsensitive gel electrophoresis for rapid detection of single-base differences in doublestranded PCR products and DNA fragments: evidence for solvent-induced bends in DNA heteroduplexes. Proc Natl Acad Sci USA 90: 10325-10329 (1993).

-Has C, Nyström A, Saeidian AH, Bruckner-Tuderman L, Uitto J: Epidermolysis bullosa: molecular pathology of connective tissue components in the cutaneous basement membrane zone. Matrix Biol 71-72:313-29 (2018).

Kiritsi D, Nyström A: Recent advances in understanding and managing epidermolysis bullosa. F1000Res 7:F1000 Faculty Rev-1097 (2018).

Kon A, Pulkkinen L, Ishida-Yamamoto A, Hashimoto I, Uitto J: Novel COL7A1 mutations in dystrophic forms of epidermolysis bullosa. J Invest Dermatol 111:534-537 (1998).

-Laimer M, Prodinger C, Bauer JW: Hereditary epidermolysis bullosa. J Dtsch Dermatol Ges 13:1125-1133 (2015).
Li AW, Prindaville B, Bateman ST, Gibson TE, Wiss K: Inpatient management of children with recessive dystrophic epidermolysis bullosa: a review. Pediatr Dermatol 34:647-655 (2017).

- Nakayama C, Fujita Y, Matsumura W, Ujiie I, Takashima S, et al: The development of induced pluripotent stem cell-derived mesenchymal stem/stromal cells from normal human and RDEB epidermal keratinocytes. J Dermatol Sci 91:301-310 (2018).

Rashidghamat E, McGrath JA: Novel and emerging therapies in the treatment of recessive dystrophic epidermolysis bullosa. Intractable Rare Dis Res 6:6-20 (2017).

-Saeidian AH, Youssefian L: First report of COL$7 A 1$ mutations in two patients with recessive dystrophic epidermolysis bullosa from Peru. Clin Exp Dermatol 43:719-722 (2018).

- Saeidian AH, Youssefian L, Moreno Trevino MG, Fortuna G, et al: Seven novel COL7A1 mutations identified in patients with recessive dystrophic epidermolysis bullosa from Mexico. Clin Exp Dermatol 43:579-584 (2018).
Vahidnezhad H, Youssefian L, Zeinali S, Saeidian $\mathrm{AH}$, Sotoudeh S, et al: Dystrophic epidermolysis bullosa: COL7A 1 mutation landscape in a multi-ethnic cohort of 152 extended families with high degree of customary consanguineous marriages. J Invest Dermatol 137:660669 (2017).

- Vahidnezhad H, Youssefian L, Saeidian AH, Uitto J: Phenotipic spectrum of epidermal bullosa: the paradigm of syndromic versus nonsyndromic skin fragility disorders. J Invest Dermatol139:522-527 (2019a).

-Vahidnezhad H, Youssefian L, Saeidian AH, Touati A, Paiouhanfar S, et al: Mutations in $P L O D 3$, encoding lysyl hydroxylase 3 , causes a complex connective tissue disorder including recessive dystrophic epidermolysis bullosa-like blistering phenotype with abnormal anchoring fibrils and type VII collagen deficiency. Matrix Biol 81:91-106 (2019b).

- Yenamandra VK, Vellarikkal SK, Chowdhury MR, Jayarajan R, Verma A, et al: Genotypephenotype correlations of dystrophic epidermolysis bullosa in India: experience from a tertiary care centre. Acta Derm Venereol 98: 873-879 (2018). 